\title{
Sensitivity Analysis of Mathematical Model of Coronavirus Disease (COVID-19) Transmission
}

\author{
Resmawan1, Lailany Yahya ${ }^{2}$ \\ 1,2 Department of Mathematics, Universitas Negeri Gorontalo \\ Jl. Prof. Dr. Ing. BJ. Habibie, Tilongkabila, Bone Bolango, Gorontalo, Indonesia \\ Email: resmawan@ung.ac.id, lailany.math@gmail.com
}

\begin{abstract}
The study was aimed to introduce a new model construction regarding the transmission of Coronavirus Disease (henceforth, COVID-19) in human population. The mathematical model was constructed by taking into consideration several epidemiology parameters that are closely identical with the real condition. The study further conducted an analysis on the model by identifying the endemicity parameters of COVID-19, i.e., the presence of disease-free equilibrium (DFE) point and basic reproductive number. The next step was to carry out sensitivity analysis to find out which parameter is the most dominant to affect the disease's endemicity. The results revealed that the parameters $\eta, \zeta_{s e}, \alpha$, and $\sigma$ in sequence showed the most dominant sensitivity index towards the basic reproductive number. Moreover, the results indicated positive index in parameters $\eta$ and $\zeta_{s e}$ that represented transmission chances during contact as well as contact rate between susceptible individuals and exposed individual. This suggests that an increase in the previous parameter value could potentially enlarge the endemicity of COVID-19. On the other hand, parameters $\alpha$ and $\sigma$, representing movement rate of exposed individuals to the quarantine class and proportion of quarantined exposed individuals, showed negative index. The numbers indicate that an increase in the parameter value could decrease the disease's endemicity. All in all, the study concludes that treatments for COVID-19 should focus on restriction of interaction between individuals and optimization of quarantine.
\end{abstract}

Keywords: Sensitivity Analysis; Mathematical Model; Coronavirus Disease; COVID-19; Basic Reproductive Number

\section{INTRODUCTION}

Coronavirus Disease (henceforth, COVID-19) has shocked many thanks to its very rapid spread. Firstly, identified to occur in Wuhan city of China, the disease has shortly become one of the main talking points as it reached the whole world and took thousands of death toll in a very short period. The disease somewhat instigates all parties to conduct active measures in finding options to the best treatments and anticipatory means to prevent damage on a much wider scale. In mathematical perspective, the concern is closely related to implementation of mathematical model to identify potential solutions.

Mathematical modelling is one of the key tools in epidemic preparation, including the COVID-19 pandemic. The system allows one to comprehend and identify the correlation between COVID-19 spread and several epidemiology parameters, conduct preparatory measures for future planning, and implement best practices of pandemic 
treatment.

Previous studies, albeit little in number, has begun to address this problem and design mathematical model for COVID-19 transmission [1][2][3][4]. The model involved accurate and effective public health interventions. On top of that, a study compared between the outbreaks of current COVID-19 and previous MERS disease that spread in Middle Eastern countries and Korea [5]. Other studies designed mathematical model that predict COVID-19 cases in different countries [6][7].

Several models proposed in previous studies has discussed that the virus started from an unknown source and eventually began to spread to human population. The virus source, further referred to as reservoir variable, is suspected to be the place of first infection-to-human case. The present study introduces a different approach on mathematical modelling to the virus transmission by also involving the epidemiology parameters; a variable that is not discussed in previous studies. Previous models have assumed that the virus transmission only occurs in interactions between individuals that have contracted the virus; differing from that, this article takes into consideration transmission cases caused by susceptible individuals and exposed individuals. It views the importance of involving such parameters, considering the number of infections that occurred in the interaction between exposed individuals yet to be detected as infected. Moreover, the model lays its emphasis on the pattern of transmission between human after the virus has become epidemic or pandemic, therefore, disregarding the reservoir variable. The model thus overlooks the process of first human infection and focuses on how the virus has spread within human-to-human interactions. In addition to that, the model employs new parameter representing death cases of COVID-19, pertaining to the fact that the virus has taken numbers of death toll. The model also takes into account cases of quarantined individuals that were identified to be exposed to the virus.

The following section elaborates construction of mathematical model in this study. Further, the article presents the research results in the form of model analysis. Within this section, the study focuses on the construction of basic reproductive number and sensitivity analysis to identify which parameter is the most sensitive to the change in basic reproductive number value. Finally, the last section proposes several conclusions to the research findings and discussion.

\section{METHODS}

In this model, the total of human population was denoted in $N(t)$ and divided into six classes: susceptible individuals $S(t)$, exposed individuals in incubation period $E(t)$, asymptotically infected individuals $A(t)$, symptomatic infected individuals $I(t)$, quarantined individuals $Q(t)$, and individuals that have recovered/remove from COVID$19 R(t)$. Therefore, the total population was stated in $N(t)=S(t)+E(t)+A(t)+I(t)+$ $Q(t)+R(t)$.

Recruitment rate of natural human natality and mortality is given the parameter $\Pi$ and $\mu$ sequentially. Susceptible individuals $(S)$ will be infected from enough contact with exposed individuals $(E)$, symptomatic infected individuals $(I)$, and asymptotically infected individuals $(A)$ in respective rate of $\eta \zeta_{s e} S E, \eta \zeta_{s i} S I$, and $\eta \zeta_{s a} S A$, in which $\eta$ is the infection probability during contact between individuals. $\zeta_{s e}, \zeta_{s i}$, and $\zeta_{s a}$ each states the contact rate between susceptible individuals $(S)$ and individual groups of $E, I$, and $A$. The parameter $\theta$ and $\sigma$ in respective order is the proportion of asymptotically infected individuals and quarantined exposed individuals, while parameter $\alpha$ states movement rate of exposed individuals to the quarantined individuals. Parameter $\omega$ and $\varpi$ each 
represent transmission rate after incubation period and status change to $I$ and $A$ class. Quarantined individuals can be transferred to the class of infected individuals with symptoms at the rate of $\varrho$ and proportion of $\varphi$. Parameter $\tau, \beta, \rho$ each states the recovery rate of infected individuals without symptoms, quarantined individuals, and infected individuals with symptoms to be transferred to recovered individuals class $I$. Further, death rate of COVID-19 in $I$ class is represented in $\delta$. below.

Schematically, the transmission pattern of COVID-19 is displayed in Figure 1

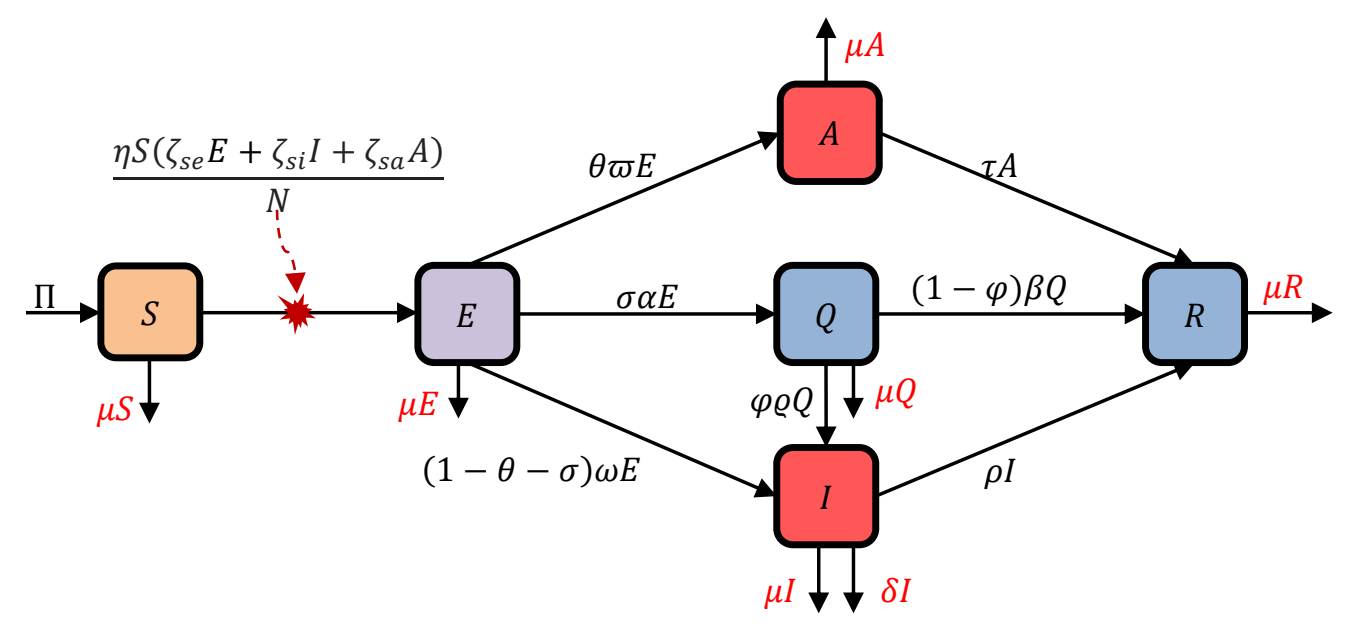

Figure 1. Compartment diagram of COVID-19 transmission

Based on the schematic diagram of virus transmission in Figure 6, the article presents model in the form of differential equation system as follows:

$\frac{d S}{d t}=\Pi-\frac{\eta S\left(\zeta_{s e} E+\zeta_{s i} I+\zeta_{s a} A\right)}{N}-\mu S$

$\frac{d E}{d t}=\frac{\eta S\left(\zeta_{s e} E+\zeta_{s i} I+\zeta_{s a} A\right)}{N}-(\theta \varpi+\sigma \alpha+\mu+\omega-\theta \omega-\sigma \omega) E$

$\frac{d A}{d t}=\theta \varpi E-(\tau+\mu) A$

$\frac{d Q}{d t}=\sigma \alpha E-(\varphi \varrho+\beta-\beta \varphi+\mu) Q$

$\frac{d I}{d t}=(1-\theta-\sigma) \omega E+\varphi \varrho Q-(\rho+\mu+\delta) I$

$\frac{d R}{d t}=\tau A+(1-\varphi) \beta Q+\rho I-\mu R$

with early condition:

$$
\begin{aligned}
& S(0)=S_{0} \geq 0, \quad E(0)=E_{0} \geq 0, \quad A(0)=A_{0} \geq 0, \\
& Q(0)=Q_{0} \geq 0, \quad I(0)=I_{0} \geq 0, \quad R(0)=R_{0} \geq 0 .
\end{aligned}
$$

Dynamics of population in total is acquired by totaling the five equations in model 
(1), resulting in:

$$
\frac{d N}{d t}=\Pi-\mu N-\delta I
$$

Positive invariant region that meets the model (1) is given by

$$
\Omega=\left\{(S(t), E(t), A(t), Q(t), I(t), R(t)) \in R_{+}^{6}: N(t) \leq \frac{\Pi}{\mu}\right\} .
$$

\section{RESULTS AND DISCUSSION}

\section{The Disease-free Equilibrium Point and The Basic Reproductive Number}

The equilibrium point is acquired at,

$$
\frac{d S}{d t}=\frac{d E}{d t}=\frac{d A}{d t}=\frac{d Q}{d t}=\frac{d I}{d t}=\frac{d R}{d t}=0
$$

therefore, the system of equation (1) indicates that the DFE point denoted by $E_{d f e}$, i.e.,

$$
E_{\text {dfe }}=\left(S_{0}, 0,0,0,0,0\right)=\left(\frac{\Pi}{\mu}, 0,0,0,0,0\right)
$$

The basic reproductive number denoted by $R_{0}$ is the expected value of infection rate per time unit. The infection occurs in a susceptible population, caused by an infected individual. Based on the equation system (1), the article generates equation that involves the classes of exposed population, infected population without symptom, and infected population with symptom, as follows:

$$
\begin{aligned}
& \frac{d E}{d t}=\frac{\eta S\left(\zeta_{s e} E+\zeta_{s i} I+\zeta_{s a} A\right)}{N}-(\theta \varpi+\sigma \alpha+\mu+\omega-\theta \omega-\sigma \omega) E \\
& \frac{d A}{d t}=\theta \varpi E-(\tau+\mu) A \\
& \frac{d Q}{d t}=\sigma \alpha E-(\varphi \varrho+\beta-\beta \varphi+\mu) Q \\
& \frac{d I}{d t}=(1-\theta-\sigma) \omega E+\varphi \varrho Q-(\rho+\mu+\delta) I
\end{aligned}
$$

Further, the basic reproductive number is calculated by referring to the job [8]. From the equation (6), the study generates matrix $\mathcal{F}$ and $\mathcal{V}$, i.e.

$$
\mathcal{F}=\left[\begin{array}{c}
\frac{\eta S\left(\zeta_{s e} E+\zeta_{s i} I+\zeta_{s a} A\right)}{N} \\
0 \\
0 \\
0
\end{array}\right] \operatorname{dan} \mathcal{V}=\left[\begin{array}{c}
(\theta \varpi+\sigma \alpha+\mu+\omega-\theta \omega-\sigma \omega) E \\
(\tau+\mu) A-\theta \varpi E \\
(\varphi \varrho+\beta-\beta \varphi+\mu) Q-\sigma \alpha E \\
(\rho+\mu+\delta) I-(1-\theta-\sigma) \omega E-\varphi \varrho Q
\end{array}\right]
$$

Using the DFE point in equation (5), Jacobian matrix $F$ and $V$ is generated, i.e. 
$F=\left[\begin{array}{cccc}\frac{\eta \zeta_{s e} S_{0}}{N} & \frac{\eta \zeta_{s a} S_{0}}{N} & 0 & \frac{\eta \zeta_{s i} S_{0}}{N} \\ 0 & 0 & 0 & 0 \\ 0 & 0 & 0 & 0 \\ 0 & 0 & 0 & 0\end{array}\right]=\left[\begin{array}{cccc}\eta \zeta_{s e} & \eta \zeta_{s a} & 0 & \eta \zeta_{s i} \\ 0 & 0 & 0 & 0 \\ 0 & 0 & 0 & 0 \\ 0 & 0 & 0 & 0\end{array}\right]$ and

$V=\left[\begin{array}{cccc}\theta \varpi+\sigma \alpha+\mu+\omega-\theta \omega-\sigma \omega & 0 & 0 & 0 \\ -\theta \varpi & \tau+\mu & 0 & 0 \\ -\sigma \alpha & 0 & \varphi \varrho+\beta-\beta \varphi+\mu & 0 \\ -(1-\theta-\sigma) \omega & 0 & -\varphi \varrho & \rho+\mu+\delta\end{array}\right]$

The basic reproductive number $R_{0}$ is acquired from the largest positive eigenvalue of next generation matrix, $K=F V^{-1}$, i.e.

$$
R_{0}=\rho\left(F V^{-1}\right), \quad \rho=\text { Dominant eigenvalue. }
$$

Therefore, it is generated that:

$$
\begin{aligned}
& R_{0}=\frac{\eta \zeta_{s e}(\tau+\mu)+\eta \zeta_{s a} \theta \varpi}{(\tau+\mu)(\mu+\omega+\theta \varpi+\alpha \sigma-\theta \omega-\sigma \omega)} \\
& +\frac{\eta \zeta_{s i} \varphi \varrho \alpha \sigma+\eta \zeta_{s i}(\omega-\sigma \omega-\theta \omega)(\mu+\beta-\beta \varphi+\varphi \varrho)}{(\mu+\delta+\rho)(\mu+\beta-\beta \varphi+\varphi \varrho)(\mu+\omega+\theta \varpi+\alpha \sigma-\theta \omega-\sigma \omega)}
\end{aligned}
$$

Further, $R_{0}$ can be stated in the form:

$$
R_{0}=R_{1}+R_{2}
$$

with

$$
\begin{aligned}
& R_{1}=\frac{\eta \zeta_{s e}(\tau+\mu)+\eta \zeta_{s a} \theta \varpi}{(\tau+\mu)(\mu+\omega+\theta \varpi+\alpha \sigma-\theta \omega-\sigma \omega)} \\
& R_{2}=\frac{\eta \zeta_{s i} \varphi \varrho \alpha \sigma+\eta \zeta_{s i}(\omega-\sigma \omega-\theta \omega)(\mu+\beta-\beta \varphi+\varphi \varrho)}{(\mu+\delta+\rho)(\mu+\beta-\beta \varphi+\varphi \varrho)(\mu+\omega+\theta \varpi+\alpha \sigma-\theta \omega-\sigma \omega)}
\end{aligned}
$$

\section{Baseline Parameter Values}

Parameter value implemented in the equation model (1) involves parameter value as a result of fitting process from several valid references based on real cases of COVID19 that has been reported and published. The complete parameter values implemented in the present study are displayed in the following Table 1.

By referring to the parameter values in Table 1 in equation (10), the study acquires estimation of basic reproductive number of $R_{0} \approx 3.097$. This indicates that every infected individual is potential to infect minimum of three new individuals.

\section{Sensivity Analysis}

To determine the most optimum approach in suppressing the number of infected individuals, one needs to identify several factors that contribute to the transmission of the 
virus and its prevalence. The first case of COVID-19 transmission closely relates to $R_{0}$ and persons exposed to COVID-19, as of $E, A, Q$, and $I$ group.

Table 1. Estimation of parameter values in COVID-19 cases

\begin{tabular}{|c|c|c|c|}
\hline Parameter & Description & Value & Source \\
\hline$\mu$ & Natural mortality rate & $3.57 \times 10^{-5}$ & Estimation \\
\hline$\eta$ & Transmission probability during contact & 0.2 & Estimation \\
\hline$\zeta_{s e}$ & $\begin{array}{l}\text { Rate of contact between susceptible individual } \\
\text { and exposed individuals }\end{array}$ & 0.09 & Estimation \\
\hline$\zeta_{s a}$ & $\begin{array}{l}\text { Rate of contact between susceptible individual } \\
\text { and asymptotically infected individuals }\end{array}$ & 0.07 & Estimation \\
\hline$\zeta_{s i}$ & $\begin{array}{l}\text { Rate of contact between susceptible individual } \\
\text { and symptomatic infected individuals }\end{array}$ & 0.05 & {$[4]$} \\
\hline$\theta$ & $\begin{array}{l}\text { Proportion of asymptotically infected } \\
\text { individuals }\end{array}$ & 0.12 & Estimation \\
\hline$\sigma$ & Proportion of quarantined exposed individuals & 0.04 & Estimation \\
\hline$\alpha$ & $\begin{array}{l}\text { Movement rate of exposed individuals to } \\
\text { quarantined individuals }\end{array}$ & 0.13266 & {$[3]$} \\
\hline$\omega$ & $\begin{array}{l}\text { Transmission rate after incubation period and } \\
\text { transferred to symptomatic infected class }\end{array}$ & 0.005 & {$[4]$} \\
\hline$\varpi$ & $\begin{array}{l}\text { Transmission rate after incubation period and } \\
\text { transferred to asymptotically infected class }\end{array}$ & 0.00048 & {$[4]$} \\
\hline$\varrho$ & $\begin{array}{l}\text { Movement rate of quarantined individual to } \\
\text { symptomatic infected individuals }\end{array}$ & 0.1259 & {$[3]$} \\
\hline$\varphi$ & $\begin{array}{l}\text { Proportion of quarantined symptomatic } \\
\text { infected individuals }\end{array}$ & 0.05 & Estimation \\
\hline$\tau$ & $\begin{array}{l}\text { Recovery rate of asymptotically infected } \\
\text { individuals and transferred to R class }\end{array}$ & 0.854302 & {$[4]$} \\
\hline$\beta$ & $\begin{array}{l}\text { Recovery rate of quarantined individuals and } \\
\text { transferred to R class }\end{array}$ & 0.11624 & {$[2]$} \\
\hline$\rho$ & $\begin{array}{l}\text { Recovery rate of symptomatic infected } \\
\text { individualsand transferred to R class }\end{array}$ & 0.33029 & {$[2]$} \\
\hline$\delta$ & Mortality rate due to COVID-19 & $1.78 \times 10^{-5}$ & {$[3]$} \\
\hline
\end{tabular}

In this section, the study calculates the sensitivity index of each parameter model that correlates with the basic reproductive numbers, $R_{0}$. This index provides information about the importance of each parameter to the model representing the transmission of COVID-19. The index is used to identify the parameter that has the most significant impact on $R_{0}$ which is later served as the intervention target. The parameter with a high impact in $R_{0}$ shows that the parameter has a dominant influence on the endemicity of COVID-19. The parameter of the sensitivity index towards the basic reproductive number is calculated using the approach similar to the one seen in [9]. Based on the explicit form of $R_{0}$ in equation (10), the study derivate the analytic expression for the sensitivity $R_{0}$ to the parameter $p$ that is

$$
C_{p}^{R_{0}}=\frac{\partial R_{0}}{\partial p} \times \frac{p}{R_{0}}
$$


Table 2. Model parameter index that is related to basic reproductive number

\begin{tabular}{|c|c|c|}
\hline Parameter & Description & Sensitivity Index \\
\hline$\eta$ & Transmission probability during contact & +1.000 \\
\hline$\zeta_{s e}$ & $\begin{array}{l}\text { Rate of contact between susceptible individual and } \\
\text { exposed individuals }\end{array}$ & +0.999 \\
\hline$\alpha$ & $\begin{array}{l}\text { Movement rate of exposed individuals to } \\
\text { quarantined individuals }\end{array}$ & -0.911 \\
\hline$\sigma$ & Proportion of quarantined exposed individuals & -0.908 \\
\hline$\omega$ & $\begin{array}{l}\text { Transmission rate after incubation period and } \\
\text { transferred to symptomatic infected class }\end{array}$ & -0.0715 \\
\hline$\varpi$ & $\begin{array}{l}\text { Transmission rate after incubation period and } \\
\text { transferred to asymptotically infected class }\end{array}$ & -0.0098 \\
\hline$\mu$ & Natural mortality rate & -0.0061 \\
\hline$\zeta_{s i}$ & $\begin{array}{l}\text { Rate of contact between susceptible individual and } \\
\text { symptomatic infected individuals }\end{array}$ & +0.0012 \\
\hline$\rho$ & $\begin{array}{l}\text { Recovery rate of symptomatic infected } \\
\text { individualsand transferred to R class }\end{array}$ & -0.0011 \\
\hline$\varphi$ & $\begin{array}{l}\text { Proportion of quarantined symptomatic infected } \\
\text { individuals }\end{array}$ & +0.0005 \\
\hline$\varrho$ & $\begin{array}{l}\text { Movement rate of quarantined individual to } \\
\text { symptomatic infected individuals }\end{array}$ & +0.0005 \\
\hline$\beta$ & $\begin{array}{l}\text { Recovery rate of quarantined individuals and } \\
\text { transferred to R class }\end{array}$ & -0.0005 \\
\hline$\theta$ & Proportion of asymptotically infected individuals & +0.0004 \\
\hline$\zeta_{s a}$ & $\begin{array}{l}\text { Rate of contact between susceptible individual and } \\
\text { asymptotically infected individuals }\end{array}$ & +0.00005 \\
\hline$\tau$ & $\begin{array}{l}\text { Recovery rate of asymptotically infected individuals } \\
\text { and transferred to R class }\end{array}$ & -0.00005 \\
\hline$\delta$ & $\begin{array}{l}\text { Mortality rate due to COVID-19 in class of } \\
\text { asymptotically infected individuals }\end{array}$ & $-6.4 \times 10^{-8}$ \\
\hline
\end{tabular}

By referring to the formulation of equation (12) and parameter value in Table 1, it is generated the sensitivity index of each parameter in the basic reproductive number $R_{0}$, presented in Table 2. As an example, the sensitivity index of $R_{0}$ towards parameter $\zeta_{s e}$ and $\alpha$ is

$$
\begin{aligned}
C_{\zeta_{s e}}^{R_{0}} & =\frac{\partial R_{0}}{\partial \zeta_{s e}} \times \frac{\zeta_{s e}}{R_{0}} \\
& =\frac{\eta}{(\mu+\omega+\theta \varpi+\alpha \sigma-\theta \omega-\sigma \omega)} \times \frac{\zeta_{s e}}{R_{0}} \\
& =\frac{\eta \zeta_{s e}}{(\mu+\omega+\theta \varpi+\alpha \sigma-\theta \omega-\sigma \omega) R_{0}} \\
& =0.999 \\
C_{\alpha}^{R_{0}} & =\frac{\partial R_{0}}{\partial_{\alpha}} \times \frac{\zeta_{\alpha}}{R_{0}}
\end{aligned}
$$




$$
\begin{aligned}
& =\frac{\eta \sigma\left(-\zeta \mathrm{se}-\frac{\zeta \mathrm{sa} \theta \varpi}{\mu+\tau}+\frac{\zeta \mathrm{si}\left(-\frac{\alpha \varrho \sigma \varphi}{\beta+\mu-\beta \varphi+\varrho \varphi}+(\theta+\sigma-1) \omega\right)}{\delta+\mu+\rho}+\frac{\zeta \operatorname{si} \varrho \varphi(\mu+\theta \varpi+\alpha \sigma+\omega-(\theta+\sigma) \omega)}{(\delta+\mu+\rho)(\beta+\mu-\beta \varphi+\varrho \varphi)}\right)}{(\mu+\theta \varpi+\alpha \sigma+\omega-(\theta+\sigma) \omega)^{2}} \times \frac{\zeta_{\alpha}}{R_{0}} \\
& =-0.911
\end{aligned}
$$

The sensitivity index in Table 2 sequentially shows the parameter with the highest sensitivity to the lowest sensitivity. In general, four parameters are significant to the changes in the basic reproductive number. Those parameters include the chances of transmission during contact $(\eta)$, the rate of contact between susceptible individuals and exposed individuals $\left(\zeta_{s e}\right)$, the movement rate of individuals to the quarantine class $(\alpha)$, and the proportion of quarantined exposed individuals $(\sigma)$. The parameter $\eta$ and $\zeta_{s e}$ has a positive sensitivity index, while the parameter $\alpha$ and $\sigma$ have a negative sensitivity index. Parameters with a positive sensitivity index represent the positive significance in the increase of basic reproductive numbers. Thereby, increasing (or decreasing) the value of the parameter, while the other parameters' value remains the same, will contribute to the increases (or decreases) in the basic reproductive numbers. Parameters with a negative sensitivity index represent the negative significance to the increase of basic reproductive numbers. In other words, increasing (or decreasing) the value of the parameter, while the other parameters' value remains the same, will contribute to the decreases (or increases) in the basic reproductive numbers.

The sensitivity index shows that contact with exposed individuals and the chance of transmission during the contact is the most dominant parameters contributing to the COVID-19 transmission. On the one hand, the proportion of quarantined exposed individuals is the parameter that suppresses the transmission of the disease the most. Since $C_{\zeta_{s e}}^{R_{0}}=+0.999$, increasing (or decreasing) the parameter $\zeta_{s e}$ by $10 \%$ will result in a increases (or decreases) of the value $R_{0}$ by $9.99 \%$. The result that $C_{\alpha}^{R_{0}}=-0.911$ signifies that increasing (or decreasing) the parameter $\alpha$ by $10 \%$ will lead to a decreases (or increases) in the value of $R_{0}$ by $9.11 \%$.

Interrupting the rate of transmission with exposed individuals and maximizing quarantine for exposed individuals and suspects are crucial. However, inadequate knowledge and lack of medical kits to detect those who have been contacted with COVID19 are now hindering the countermeasures for combating the disease. It can be said that there is no strong evidence to determine those who have been contacted with COVID-19 and those who are not carrying the virus. Due to the situations, preventive measures, such as social and physical distancing, are still the only option to halt the transmission of COVID-19.

\section{CONCLUSIONS}

The index of the sensitivity parameter shows that the parameter $\eta$ and $\zeta_{s e}$ that represents the chance of transmission during the contact and the rate of contact between the susceptible individuals and exposed individuals have the most dominant sensitivity to raise the endemicity of COVID-19. On the other hand, the parameter $\alpha$ and $\sigma$, which represent the movement rate of exposed individuals to the quarantine and the proportion of quarantined exposed individuals, are the ones that can decrease the endemicity of COVID-19 the most. The effectiveness of the measurement against COVID-19, therefore, can be assured by suppressing the interaction rate between susceptible individuals and 
exposed individuals and maximizing treatment in the quarantine for those who are infected with the disease. All these attempts can be optimized by adhering to the health protocol, such as by keeping space between oneself and the other, or well-known as social or physical distancing.

\section{ACKNOWLEDGMENTS}

This study is financially supported by LPPM Universitas Negeri Gorontalo, as stated in the Decree Number B/105/UN47.D1/PT.01.03/2020.

\section{REFERENCES}

[1] B. Tang et al., "Estimation of the Transmission Risk of the 2019-nCoV and Its Implication for Public Health Interventions," Journal of Clinical Medicine, vol. 9, no. 2, p. 462, 2020, doi: $10.3390 /$ jcm 9020462 .

[2] B. Tang et al., "The effectiveness of quarantine and isolation determine the trend of the COVID-19 epidemics in the final phase of the current outbreak in China," Int J Infect Dis, 2020, doi: 10.1016/j.ijid.2020.03.018.

[3] B. Tang, N. L. Bragazzi, Q. Li, S. Tang, Y. Xiao, and J. Wu, "An updated estimation of the risk of transmission of the novel coronavirus (2019-nCov)," Infectious Disease Modelling, vol. 5, pp. 248-255, 2020, doi: 10.1016/j.idm.2020.02.001.

[4] M. A. Khan and A. Atangana, "Modeling the dynamics of novel coronavirus (2019nCov) with fractional derivative," Alexandria Engineering Journal, 2020, doi: 10.1016/j.aej.2020.02.033.

[5] T. M. Chen, J. Rui, Q. P. Wang, Z. Y. Zhao, J. A. Cui, and L. Yin, "A mathematical model for simulating the phase-based transmissibility of a novel coronavirus," Infectious Diseases of Poverty, vol. 9, no. 1, pp. 1-8, 2020, doi: 10.1186/s40249-020-00640-3.

[6] H. Sun, Y. Qiu, H. Yan, Y. Huang, Y. Zhu, and S. X. Chen, "Tracking and Predicting COVID-19 Epidemic in China Mainland," medRxiv, no. February, p. 2020.02.17.20024257, 2020, doi: 10.1101/2020.02.17.20024257.

[7] T. Kuniya, "Prediction of the Epidemic Peak of Coronavirus Disease in Japan, 2020," Journal of Clinical Medicine, vol. 9, no. 3, p. 789, 2020, doi: 10.3390/jcm9030789.

[8] P. van den Driessche and J. Watmough, "Further Notes on the Basic Reproduction Number," in Mathematical Epidemiology, Victoria: Springer, 1945.

[9] N. Chitnis, J. M. Hyman, and J. M. Cushing, "Determining Important Parameters in the Spread of Malaria Through the Sensitivity Analysis of a Mathematical Model," Bulletin of Mathematical Biology, vol. 70, no. 5, pp. 1272-1296, Jul. 2008, doi: 10.1007/s11538-008-9299-0. 